\title{
Ranking fuzzy numbers using preference ratio: A utility function approach
}

\author{
Soheil Sadi-Nezhad $^{\mathrm{a}^{*}}$ and Parisa Shahnazari-Shahrezaei ${ }^{\mathrm{b}}$
}

${ }^{a}$ Department of Industrial Engineering, Science and Research Branch, Islamic Azad University, Tehran, Iran

${ }^{b}$ Department of Industrial Engineering, Firoozkooh Branch, Islamic Azad University, Firoozkooh, Iran

\section{CHRON I C LE ABSTRACT}

Article history:

Received October 2, 2012

Received in Revised Format

March 12, 2013

Accepted March 14, 2013

Available online

March 152013

Keywords:

Fuzzy ranking

Preference ratio

Utility function

Fuzzy number

\begin{abstract}
Ranking fuzzy numbers is one of the most important phases in any decision making process in fuzzy environments. In most cases, we deal with fuzzy numbers in the field of evaluating alternatives with fuzzy information or linguistic variables. This paper investigates ranking fuzzy numbers using the concept of preference ratio, introduces the weakness of this method, and proposes a new approach that overcomes the shortcoming of existing method. The proposed approach which is based on the concept of utility function takes the opinion of DM for ranking fuzzy numbers into account.
\end{abstract}

\section{Introduction}

Fuzzy set theory (Zadeh, 1965) has been applied to many areas, which need to manage uncertain and vague data. Such areas include approximate reasoning, decision making, data analysis, artificial intelligence, socioeconomic systems, optimization, control, and so on, in which ranking the fuzzy numbers is of importance. In order to rank fuzzy numbers, one fuzzy number should be evaluated and compared with others, but this process may be troublesome. Since fuzzy numbers are represented by possibility distributions, they can overlap with each other and thus it is difficult to clearly determine whether one fuzzy number is larger or smaller than the other one. Various techniques are applied to compare the fuzzy numbers. Some studies (Abbasbandy \& Asadi, 2006; Huijun \& Jianjun, 2006; Wang et al., 2006; Asady \& Zendehnam, 2007; Asady, 2010) introduced a ranking function to map fuzzy numbers to real numbers and then applied usual ranking methods. Other researchers (Delgado et al., 1988; Mabuchi, 1988; Tseng \& Klein, 1989; Modarres \& Sadi-Nezhad, 2001) defined a comparison function that maps two fuzzy numbers to a real number which denotes the domination degree of one fuzzy number to the other one.

* Corresponding author.

E-mail addresses: sadinejad@hotmail.com (S. Sadi-Nezhad)

C 2013 Growing Science Ltd. All rights reserved.

doi: $10.5267 /$ j.ds1.2013.03.002 
The majority of approaches are biased on the possibility notion and/or the probability measure of fuzzy events concept (Lee \& Li, 1988). Several authors (Lee \& Li, 1988; Kim \& Park, 1990; Choobineh \& Li, 1993; Fortemps \& Roubens, 1996) surveyed and compared some of these approaches using the same set of examples, as provided by Bortolan and Degani (1985). Chen and Hwang (1992) comprehensively examined the existing approaches and indicated some conditions that illogically occur among them. Moreover, Shieh (2007) offered a pair of formulas to determine the centroid of a fuzzy number. These formulas can be applied to rank all fuzzy numbers. Wang and Lee (2008) proposed a revised method for ranking fuzzy numbers with an area between their centroid and original points in order to modify Chu and Tsao's method (Chu \& Tsao, 2002).

$\mathrm{Ma}$ and $\mathrm{Li}$ (2008) presented a novel fuzzy ranking method with range reduction techniques and pairwise preference comparisons. Chen and Tang (2008) ranked non-normal p-norm trapezoidal fuzzy numbers with integral value proposed by Liou and Wang (1992). Liu et al. (2008) provided a new fuzzy ranking procedure which was consistent with the preference of investors. Their method had more potential to distinguish the order of two fuzzy numbers. Lee and Chen (2008) demonstrated a method for ranking trapezoidal fuzzy numbers on the basis of their shapes and deviations and applied this method to present a new fuzzy risk analysis algorithm. Chen and Chen (2009) developed a new method for ranking generalized fuzzy numbers regarding the defuzzified values, heights and spreads. They also proposed a fuzzy risk analysis algorithm based on their ranking method.

Chen and Wang (2009) presented an innovative method for ranking fuzzy numbers using the $\alpha$-cuts, belief feature and signal/noise ratios, and applied it to a new approach for fuzzy risk analysis. Wang and Luo (2009) suggested an alternative ranking approach for fuzzy numbers based on positive and negative ideal points. They overcame the drawback of a popular method proposed by Chen (1985). Wang et al. (2009) introduced an approach for ranking L-R fuzzy numbers based on deviation degree in order to overpower the limitations of existing methods and simplify the computational procedures. Abbasbandy and Hajjari (2009) recommended a novel method for ranking the trapezoidal fuzzy numbers based on the left and right spreads at some $\alpha$-levels. Wang (2009) reexamined two common approaches for ranking fuzzy numbers, centroid defuzzification and the maximizing set and minimizing set methods, with indefinite membership functions and available $\alpha$ level sets.

Some of the existing approaches are difficult to understand and suffer from different plights, e.g., lack of discrimination, producing counterintuitive orderings, and ultimately resulting in inconsistent orderings if a new fuzzy number is added, and some of them have high complexity and need cumbersome computational efforts (Chen \& Klein, 1997; Chen \& Klein, 1997). Although, most of approaches should acquire membership functions of fuzzy numbers before the ranking is performed, but this matter may be impossible in real applications. Furthermore, accuracy and efficiency should be of priority concern in the ranking process if ranking a large amount of fuzzy numbers is necessary.

The rest of this paper is organized as follows. Section 2 deals with an introduction to ranking fuzzy numbers by "Preference Ratio Method" (Modarres \& Sadi-Nezhad, 2001) and discussion on the weakness of this method. A novel comparison method based on utility function is presented in Section 3. Some numerical examples are solved by the proposed method in Section 4. Finally, Section 5 sums up concluding remarks.

\section{Introduction to fuzzy ranking by preference ratio method}

This method, which compares fuzzy numbers relatively, was proposed by Modarres and Sadi-Nezhad (2001). In this method, fuzzy numbers are evaluated point by point and ranked at each point. Subsequently, total preference, which is relative rather than absolute, over all points is calculated. Assume the objective is to rank $I$ fuzzy numbers. Consider $N_{i}$ be the $i^{\text {th }}$ one defined over a real domain $S_{i} \subset R$ and is specified by a membership function $\left(\mu_{N i}(x), x \in S_{i}\right)$ with $\mu_{N i} \in[0,1]$. Let $S_{i}$ be the 
support of $N_{i}$, i.e., $S_{i}=\left\{x, \mu_{N i}(x)>0\right\}$, and $\Omega=\cup_{i=1}^{I} \mathrm{Si}$, then $\Omega$ is union of the support of all fuzzy numbers, i.e., fuzzy numbers are ranked over $\Omega$. Since ranking the fuzzy numbers with disjoint spans is clear, it is assumed the fuzzy numbers have joint spans.

A fuzzy number is evaluated at each point $\alpha \in \Omega$ by Eq. (1) which is called Preference Function:

$G(\alpha)=\frac{\int_{\alpha}^{U} \mu(x) d(x)}{\int_{L}^{U} \mu(x) d(x)}$

where, $\mu(x)$ is the membership function of fuzzy number, $L=\min \{x: x \in \Omega\}$ and $U=\max \{x: x \in \Omega\}$. This function has the same definition as $1-F(\alpha)$ in probability theory, where $F(\alpha)=P[X \leq \alpha]$ is the distribution function. At $\alpha \in \Omega$, let $p(\alpha)=i$ indicates the $i^{\text {th }}$ fuzzy number which is the most preferred one. According to Eq. (2):

$p(\alpha)=i, \quad$ if $\quad G i(\alpha)=\max \{G j(\alpha), j \in I\}$

where, $G_{j}(\alpha)$ is the preference function of $j^{\text {th }}$ fuzzy number. Eq. (3) expresses the set of points at which the $i^{\text {th }}$ number is ranked number one:

$\Omega i=\{\alpha \in \Omega, p(\alpha)=i\}$

Definition 1. For the $i^{\text {th }}$ fuzzy number, the Preference Ratio $R(i)$ which is defined by Eq. (4) is the percentage of $\Omega$ that the $i^{\text {th }}$ fuzzy number is the most preferred one:

$R(i)=\frac{|\Omega i|}{|\Omega|}$,

where, $|\Omega \mathrm{i}|$ and $|\Omega|$ are the lengths of real sets $\Omega \mathrm{i}$ and $\Omega$, respectively.

\subsection{Preference ratio for continuous numbers}

In order to determine preference ratio for fuzzy numbers $A_{i}, i=1, \ldots, n$, it is necessary to calculate $G_{i}(\alpha)$ from Eq. (1) at each point $\alpha \in \Omega$ first, and for every $i=1, \ldots, n$. Afterwards, $\Omega_{i}$ and $R(i)$ are calculated from Eq. (3) and Eq. (4), respectively. Algorithm1 which is on the basis of search technique is applicable for continuous fuzzy numbers and obtains the results relatively fast (Modarres \& Sadi-Nezhad, 2005).

\section{Algorithm 1}

Assume $A_{1}$ and $A_{2}$ be two fuzzy numbers with continuous membership functions and joint spans. Hence, $G_{j}(\alpha)$ is calculated for $j=1,2$ at each interval of $e$ in $\Omega$ using Eq. (5) and Eq. (6):

$e=\frac{U-L}{1000}$

and,

$L=\min \{x: x \in \Omega\}$ and $U=\max \{x: x \in \Omega\}$

Obviously, more accurate results are acquired by setting smaller intervals.

Step 1. Read $A_{1}$ and $A_{2}$ with their membership functions of $\mu_{A_{1}}$ and $\mu_{A_{2}}$, 
Step 2. Determine $L$ and $U$ as well as $e$ from Eq. (5). Set $\alpha=L+e, \mathrm{R}\left(A_{1}\right)=0$ and $\mathrm{R}\left(A_{2}\right)=0$,

Step 3. Calculate $G_{j}(\alpha)$, for $j=1,2$. If $G_{l}(\alpha)>G_{2}(\alpha)$, then set $\mathrm{R}\left(A_{1}\right)=\mathrm{R}\left(A_{1}\right)+e$ and go to Step 4. If $G_{1}(\alpha)<G_{2}(\alpha)$, then set $\mathrm{R}\left(A_{2}\right)=\mathrm{R}\left(A_{2}\right)+e$ and go to Step 4. If $G_{1}(\alpha)=G_{2}(\alpha)$, then set $\mathrm{R}\left(A_{1}\right)=$ $\mathrm{R}\left(A_{1}\right)+e / 2$ and $\mathrm{R}\left(A_{2}\right)=\mathrm{R}\left(A_{2}\right)+e / 2$ and go to Step 4 ,

Step 4. Set $\alpha=\alpha+e$. If $\alpha<U$, then go to Step 3. Otherwise stop.

\subsection{Weakness of preference ratio method}

If the objective is to rank more than two fuzzy numbers, it is not practical to compare them pair-wise; especially in the case that there are too many fuzzy numbers. In this case, preference ratio is an efficient ranking method. But by applying preference ratio, it will be seen the syllogism is not held as a general rule. This problem will be illustrated by Example 1.

Example 1. Consider three arbitrary triangular fuzzy numbers $A, B$ and $C$ according to Fig. $1: A=$ $(0,0,10), B=(4.9,4.9,4.9)$ and $C=(0,9,9)$. First, compare two fuzzy numbers $A$ and $C$. It is realized that $A$ is less than $C$ by preference ratio:

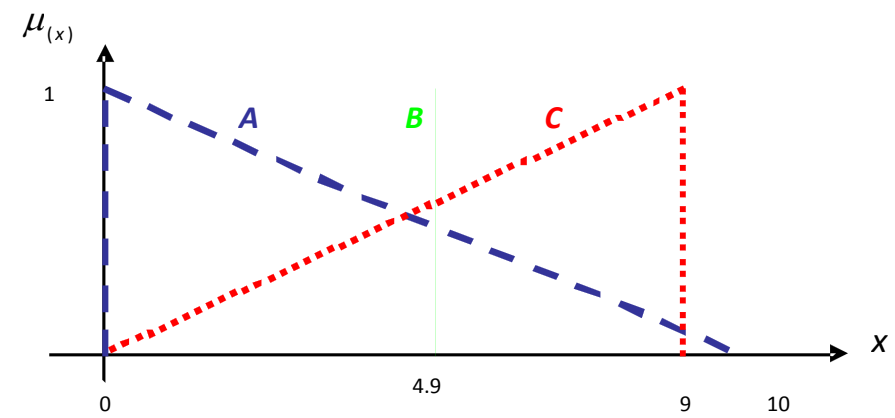

Fig. 1. Comparison of fuzzy numbers $A=(0,0,10), B=(4.9,4.9,4.9)$ and $C=(0,9,9)$

$$
R(A)=0.105, \quad R(C)=0.895 \quad===>\quad A<C
$$

Then, compare two fuzzy numbers $B$ and $C$. As a result, $C$ is less than $B$ by preference ratio:

$$
R(B)=0.544, \quad R(C)=0.456 \quad===>\quad C<B
$$

Since $A$ is less than $C$ and $C$ is less than $B$, according to syllogism rule, $A$ must be less than $B$. By applying preference ratio to compare fuzzy numbers $A$ and $B$, the following result is obtained:

$$
R(A)=0.51, \quad R(B)=0.49 \quad===>\quad A>B
$$

Contrary to general expectation, preference ratio of $A$ is greater than preference ratio of $B$. Accordingly, the syllogism rule is violated. To avoidance of this violation in multiple attribute decision making (MADM) problems, Modarres and Sadi-nezhad (2005) propounded an idea without any referring to this contradiction. They compared each fuzzy number with a specified one as a benchmark. By applying preference ratio concept, comparison of each pair of fuzzy numbers ends up in a crisp ratio. Therefore, they changed the scale of span of fuzzy numbers. In other words, they multiplied fuzzy numbers by a real number $\mathrm{k}$ and expressed the concepts of equivalence by preference ratio and equivalence multiplier (Modarres \& Sadi-Nezhad, 2005). 
This concept motivated other researchers to employ this ranking method in their investigations (SadiNezhad \& Ghaleh Assadi, 2008, Sadi-Nezhad \& Khalili Damghani, 2010). By means of this manipulation, they found out that $A<C<B$, and this is contrary to the results of Example 1. It should be noted, the syllogism rule is not propounded in this technique, because all fuzzy numbers are compared with a benchmark.

\section{Utility value of different points in decision space}

In decision theory, utility is a measure of the desirability of consequences of action's courses which applies to decision making under risk, namely under uncertainty with known probabilities. The concept of utility applies to both single-attribute and multi-attribute consequences. Based on fundamental assumption in utility theory, DM always chooses the alternative for which the expected value of utility (expected utility) is maximum. If this assumption is accepted, utility theory can be used to predict or prescribe the choice that DM will make or should make among the available alternatives. For this purpose, a utility has to be assigned to any possible (and mutually exclusive) consequences of each alternative. A utility function is the rule by which this assignment is done and depends on the preferences of individual DM. In spite of the Preference Ratio method that all points have the same utility for DM, in utility theory, each point of decision space can have its own relevant utility value. Therefore, this matter is considered in the proposed comparison method.

\subsection{A generalized comparison method based on utility function}

In the proposed method by Modarres and Sadi-Nezhad (2001), each fuzzy number is evaluated by preference function. In this paper, utility function is considered in the calculations of proposed comparison method.

Definition 2. $\mathrm{U}(\mathrm{x})$ for $x \in \Omega$ is the utility function which is related to DM's perception about the utility of each point in decision space. For $i^{\text {th }}$ fuzzy number, $S(i)$ denotes the $i^{\text {th }}$ fuzzy number's score according to Eq. (7):

$S(i)=\int_{x \in \Omega_{i}} U(x) d x$

and relational preference is calculated by Eq. (8):

$R(i)=\frac{S(i)}{\int_{x \in \Omega} U(x) d x}$

Utility function can have various forms, such as: linear, nonlinear, uniform and the like. Also, Utility function can consider maximum membership value and so on. If utility function has uniform distribution $(U(x)=a$; $a$ is a constant value) in Eq. (7) and Eq. (8), the primary method proposed by Modarres and Sadi-Nezhad (2001) will be obtained:

$S(i)=\int_{x \in \Omega_{i}} U(x) d x=\int_{x \in \Omega i} a d x=a \Omega_{i}$

and,

$R(i)=\frac{S(i)}{\int_{x \in \Omega} U(x) d x}=\frac{a \Omega_{i}}{\int_{x \in \Omega} a d x}=\frac{a \Omega_{i}}{a \Omega}=\frac{\Omega_{i}}{\Omega}$

The result acquired by Eq. (9) and Eq. (10) conforms to Eq. (4). Now, the above-mentioned fuzzy ranking method is generalized for more than two fuzzy numbers. Hence, fuzzy numbers are compared pair-wise by preference ratio method and turning points (the points at which preference changes from one fuzzy number to the other one) are calculated. Then, the utility value of each point is added to computations. 
Proposed algorithm: preference ratio based on utility function algorithm

Let $A_{i}=\left(L_{i}, M_{i}, U_{i}\right)$ for $i=1, \ldots, m$ be triangle fuzzy numbers. The objective is to compare these fuzzy numbers pair-wise by preference ratio method and utility function, and then rank them in increasing or decreasing order.

Initialization Step. $i=1$ and $j=1$.

Step 1. If $j=m$, set $i=i+1$ and $j=i$. If $i>m$-1, then stop and rank the fuzzy numbers on the basis of existing results.

Step 2. If $j<m$, then set $j=j+1$.

Step 3. Let two fuzzy numbers $A_{i}=\left(L_{i}, M_{i}, U_{i}\right)$ and $A_{j}=\left(L_{j}, M_{j}, U_{j}\right)$, and obtain $L, M, \bar{M}$, and $\bar{U}$ according to Eq. (11):

$$
L_{-}=\min \left\{L_{i}, L_{j}\right\}, M_{-}=\min \left\{M_{i}, M_{j}\right\}, \bar{M}=\max \left\{M_{i}, M_{j}\right\}, \bar{U}=\max \left\{U_{i}, U_{j}\right\}
$$

Step 4. Calculate feasible turning points ( $\alpha$ s) using preference ratio method as Eqs. (12-14):

a) In the interval $\left[L_{-}, M_{-}\right], \frac{\int_{L_{i}}^{\alpha} \mu_{A_{i}}(x) d x}{\int_{L_{i}}^{U_{i}} \mu_{A_{i}}(x) d x}=\frac{\int_{L_{j}}^{\alpha} \mu_{A_{j}}(x) d x}{\int_{L_{j}}^{U_{j}} \mu_{A_{j}}(x) d x}$

b) In the interval $[M, \bar{M}]$,

$$
S_{\left[L_{i}, M\right]}+\int_{-}^{\alpha} \mu_{A_{i}}(x) d x \quad S_{\left[L_{j}, M\right]}+\int_{-}^{\alpha} \mu_{A_{j}}(x) d x
$$

c) In the interval $[\bar{M}, \bar{U}]$,

$$
\frac{-}{\int_{L_{i}}^{U_{i}} \mu_{A_{i}}(x) d x}=\frac{-\int_{L_{j}}^{U_{j}} \mu_{A_{j}}(x) d x}{s}
$$

$$
\frac{S_{\left[L_{i} \bar{M}\right]}+\int_{\bar{M}}^{\alpha} \mu_{A_{i}}(x) d x}{\int_{L_{i}}^{U_{i}} \mu_{A_{i}}(x) d x}=\frac{S_{\left[L_{j}, \bar{M}\right]}+\int_{\bar{M}}^{\alpha} \mu_{A_{j}}(x) d x}{\int_{L_{j}}^{U_{j}} \mu_{A_{j}}(x) d x}
$$

If no feasible turning point exists, it will be obvious one of these two fuzzy numbers is greater than the other one in all points and go to Step 1.

Step 5. Compute each fuzzy number's score $\left(S\left(A_{i}\right)\right.$ and $\left.S\left(A_{j}\right)\right)$ and relational preference $\left(R\left(A_{i}\right)\right.$ and $R\left(A_{j}\right)$ ) considering utility value of each point and using Eq. (7) and Eq. (8):

$$
\begin{array}{ll}
S\left(A_{i}\right)=\int_{x \in \Omega_{A_{i}}} U(x) d x, & R\left(A_{i}\right)=\frac{S(A i)}{\int_{x \in \Omega} U(x) d x} \\
S\left(A_{j}\right)=\int_{x \in \Omega_{A_{j}}} U(x) d x, & R\left(A_{j}\right)=\frac{S(A j)}{\int_{x \in \Omega} U(x) d x}
\end{array}
$$

Based on the results of Eq. (15) and Eq. (16), if $R\left(A_{i}\right)>R\left(A_{j}\right)$, then $A_{i}>A_{j}$ and go to Step 1 . Else, $A_{j}>A_{i}$ and go to Step 1. 


\subsection{From linear to sigmoidal utility function}

As it was mentioned before, utility function can have various forms, such as: uniform, linear, nonlinear and so on. As a matter of fact, utility function is formed by determining the values of different points by DM. It can vary from linear to sigmoidal function.

Let $A$ and $B$ be two fuzzy numbers which have to be compared by preference ratio method and $\Omega$ is union of the support of $A$ and $B$. Also, suppose $a$ and $b$ are the lower and upper bounds of $\Omega$, respectively. An ascending or descending linear utility function $U(x)$ is defined by drawing a straight line from point $a$ to point $b$. Eq. (17) shows the values of $U(x)$ at extreme points for both cases:

$\begin{cases}U(a)=0, U(b)=1 & ; \text { ascending linear utility function } \\ U(a)=1, U(b)=0 & ; \text { descending linear utility function }\end{cases}$

and these linear utility functions are determined by Eq. (18):

$$
\begin{cases}\mathrm{U}(\mathrm{x})=\frac{\mathrm{x}-\mathrm{a}}{\mathrm{b}-\mathrm{a}} ; & \text { ascending linear utility function } \\ \mathrm{U}(\mathrm{x})=\frac{\mathrm{x}-\mathrm{b}}{\mathrm{a}-\mathrm{b}} ; & \text { descending linear utility function }\end{cases}
$$

Also, Eq. (19) displays an ascending or descending sigmoidal function:

$U(x ; \alpha, \beta)=\frac{1}{1+e^{-\alpha(x-\beta)}}$

Example 2. Two fuzzy numbers $A=(0,10,20)$ and $B=(2,7,9)$ are compared using ascending and descending linear utility functions and the proposed algorithm. From Step 3:

$L=0, \quad M=7, \quad \bar{M}=10, \quad \bar{U}=20$

Turning points are acquired according to Step 4. First, these fuzzy numbers are compared in the interval $[0,7]$ :

$$
\frac{\int_{0}^{\alpha} \mu_{A}(x) d x}{\int_{0}^{20} \mu_{A}(x) d x}=\frac{\int_{2}^{\alpha} \mu_{B}(x) d x}{\int_{2}^{9} \mu_{B}(x) d x}==>\frac{\int_{0}^{\alpha} \frac{x}{10} d x}{\int_{0}^{10} \frac{x}{10} d x+\int_{10}^{20}\left(2-\frac{x}{10}\right) d x}=\frac{\int_{2}^{\alpha}\left(\frac{x}{5}-\frac{2}{5}\right) d x}{\int_{2}^{7}\left(\frac{x}{5}-\frac{2}{5}\right) d x+\int_{7}^{9}\left(-\frac{x}{2}+\frac{9}{2}\right) d x}==>\alpha=3.44
$$

Then, the comparison is made in the interval $[7,10]$ :

$$
\frac{\int_{0}^{\alpha} \mu_{A}(x) d x}{\int_{0}^{20} \mu_{A}(x) d x}=\frac{\int_{2}^{\alpha} \mu_{B}(x) d x}{\int_{2}^{9} \mu_{B}(x) d x}==>\frac{2.45+\int_{7}^{\alpha} \frac{x}{10} d x}{\int_{0}^{10} \frac{x}{10} d x+\int_{10}^{20}\left(2-\frac{x}{10}\right) d x}=\frac{2.5+\int_{7}^{\alpha}\left(-\frac{x}{2}+\frac{9}{2}\right) d x}{\int_{2}^{7}\left(\frac{x}{5}-\frac{2}{5}\right) d x+\int_{7}^{9}\left(-\frac{x}{2}+\frac{9}{2}\right) d x}
$$

$\therefore$ There is no turning point in this interval. 
Fig. 2 clearly shows $A$ is greater than $B$ in the interval [10,20]. As a result, $B$ is greater than $A$ from 0 to 3.44. Afterwards, $A$ is greater than $B$. So, turning point is 3.44. In conformity with Step 5 of the proposed algorithm, relational preference of $A$ and $B$ should be computed regarding the utility value of each point.

a) If an ascending linear utility function is considered like Fig. 2, the utility function will be equal to $U(x)=\frac{x}{20}$ and preference ratios will be computed as follows:

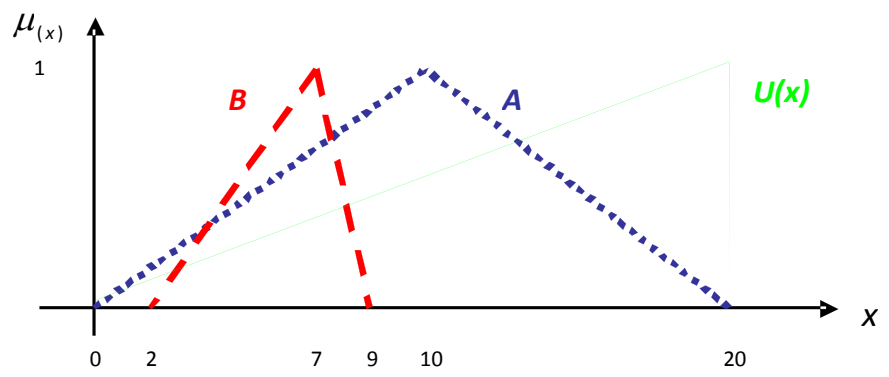

Fig. 2. Comparison of fuzzy numbers $A=(0,10,20)$ and $B=(2,7,9)$ considering an ascending linear utility function

$R(A)=\frac{\int_{3.44}^{20} \frac{x}{20} d x}{\int_{0}^{20} \frac{x}{20} d x}=0.970416, \quad R(B)=\frac{\int_{0}^{3.44} \frac{x}{20} d x}{\int_{0}^{20} \frac{x}{20} d x}=0.029584==\Rightarrow A>B$

b) Now, if a descending linear utility function is considered like Fig. 3, the utility function will be equal to $U(x)=1-\frac{x}{20}$ and preference ratios will be computed as follows:

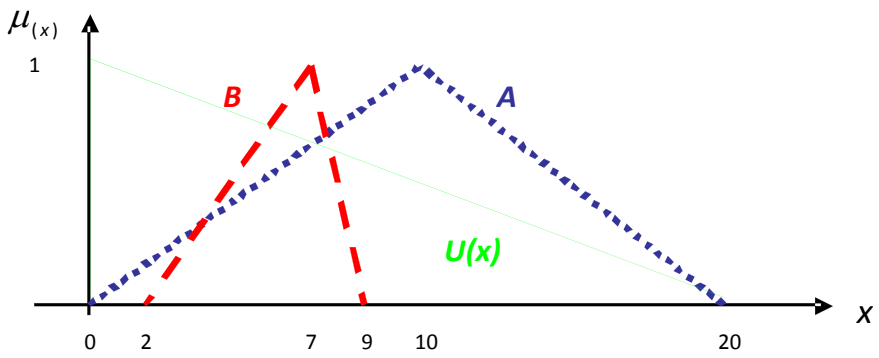

Fig. 3. Comparison of fuzzy numbers $A=(0,10,20)$ and $B=(2,7,9)$ considering a descending linear utility function

$R(A)=\frac{\int_{3.44}^{20}\left(1-\frac{x}{20}\right) d x}{\int_{0}^{20}\left(1-\frac{x}{20}\right) d x}=0.685584, R(B)=\frac{\int_{0}^{3.44}\left(1-\frac{x}{20}\right) d x}{\int_{0}^{20}\left(1-\frac{x}{20}\right) d x}=0.314416==>A>B$

Although, $A$ is greater than $B$ in both cases, but the greatness ratio of $A$ is not equal. Sometimes, determining an appropriate utility function is difficult for DM. Under these circumstances, DM's utility function can vary from an ascending to a descending linear (Fig. 4.a) or nonlinear function (Fig. 4.b). Consequently, relational preferences resulted from these utility functions form a triangle fuzzy number (TFN) as Fig. 4.c. 


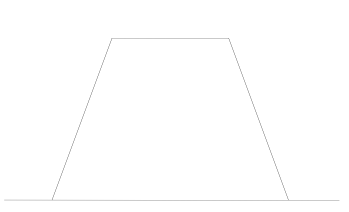

(a)

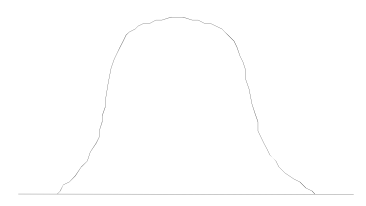

(b)

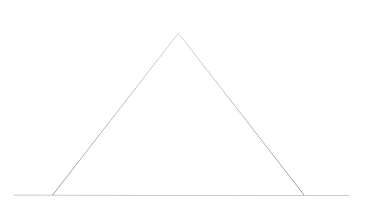

(c)

Fig. 4.a Variations of a linear utility function in DM's mind Fig. 4.b Variations of a nonlinear utility function in DM's mind

Fig. 4.c Relational preferences resulted from variations of utility function in DM's mind

This reality is illustrated by some examples presented in Table 1 . In all cases, $A$ is greater than $B$ and utility function have various forms including ascending, constant, and descending linear utility function. As the results show, when DM is doubtful for determining utility function, he/she can express his/her relational preferences by a triangle fuzzy number (Table 2), but relational preferences may be increased or decreased by enlarging or lessening the utility. Since increasing or decreasing behavior of relational preferences is not predictable, a general criterion can be acquired as Lemma 1 .

\section{Table 1}

Relational preferences resulted from different linear utility functions

\begin{tabular}{lllllllll}
\hline$A$ & $B$ & $\begin{array}{l}\text { Turning } \\
\text { Point(s) }\end{array}$ & $\begin{array}{l}R(A) \text { with } \\
\text { ascending } \\
\text { linear utility } \\
\text { function }\end{array}$ & $\begin{array}{l}R(A) \text { with } \\
\text { constant } \\
\text { utility } \\
\text { function }\end{array}$ & $\begin{array}{l}R(A) \text { with } \\
\text { descending } \\
\text { linear utility } \\
\text { function }\end{array}$ & $\begin{array}{l}R(B) \text { with } \\
\text { ascending } \\
\text { linear utility } \\
\text { function }\end{array}$ & $\begin{array}{l}R(B) \text { with } \\
\text { constant } \\
\text { utility } \\
\text { function }\end{array}$ & $\begin{array}{l}R(B) \text { with } \\
\text { descending } \\
\text { linear utility } \\
\text { function }\end{array}$ \\
\hline$(7,10,13)$ & $(1,5,9)$ & --- & 1 & 1 & 1 & 0 & 0 & 0 \\
$(2,3,9)$ & $(1,5,8)$ & $3,5.5858$ & 0.7340 & 0.6768 & 0.6200 & 0.2660 & 0.3232 & 0.3800 \\
$(1,5,8)$ & $(2,3,6)$ & 2.607625 & 0.9473 & 0.7703 & 0.5934 & 0.0527 & 0.2297 & 0.4066 \\
$(3,7,9)$ & $(1,5,10)$ & 7.932 & 0.5932 & 0.7702 & 0.9472 & 0.4068 & 0.2298 & 0.0528 \\
\hline
\end{tabular}

Table 2

Relational preferences expressed by TFNs

\begin{tabular}{llll}
\hline$A$ & $B$ & Relational Preferences of $A$ & Relational Preferences of $B$ \\
\hline$(7,10,13)$ & $(1,5,9)$ & $(1,1,1)$ & $(0,0,0)$ \\
$(2,3,9)$ & $(1,5,8)$ & $(0.6200,0.6768,0.7340)$ & $(0.2660,0.3232,0.3800)$ \\
$(1,5,8)$ & $(2,3,6)$ & $(0.5934,0.7703,0.9473)$ & $(0.0527,0.2297,0.4066)$ \\
$(3,7,9)$ & $(1,5,10)$ & $(0.5932,0.7702,0.9472)$ & $(0.0528,0.2298,0.4068)$ \\
\hline
\end{tabular}

Lemma 1: If $A$ is relatively greater than $B$ over all points, the form of utility function will not be important and anyway, $A$ will be relatively greater than $B$.

\section{Numerical examples}

a. Consider the fuzzy numbers $A=(0,0,10), B=(4.9,4.9,4.9)$ and $C=(0,9,9)$ propounded in Example 1. These fuzzy numbers are compared based on descending, constant and ascending linear utility functions by applying 'preference ratio based on utility function"' algorithm. $U_{1}(x), U_{2}(x)$ and $U_{3}(x)$ are descending, constant and ascending linear utility functions, respectively.

\section{a.1. Pair-wise comparison between $A$ and $B$}

Fig. 5 compares fuzzy numbers $A$ and $B$. Turning points should be determined first. Obviously, $B$ is greater than $A$ from 0 to 4.9 and afterwards, $A$ is greater than $B$. 


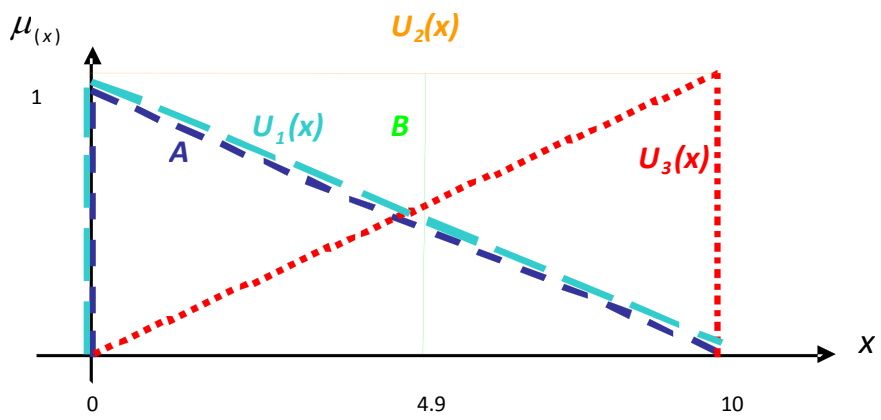

Fig. 5. Comparison of fuzzy numbers $A=(0,0,10)$ and $B=(4.9,4.9,4.9)$ based on descending, constant and ascending linear utility functions

The preference ratios are calculated using different linear utility functions as follows.

\begin{tabular}{|c|c|c|c|}
\hline$U_{1}(x):$ & 0.2601 & $R(B)=0.7399$ & $==>$ \\
\hline$U_{2}($. & $R(A)=0.5100$ & $R(B)=0.4900$ & $===>$ \\
\hline$U_{3}(x)$ & $R(A)=0.7599$ & $R(B)=0.2401$ & in \\
\hline
\end{tabular}

a.2. Pair-wise comparison between $B$ and $C$

Fig. 6 compares fuzzy numbers B and C. Clearly, B is greater than C from 0 to 4.9 and afterwards, C is greater than $\mathrm{B}$.

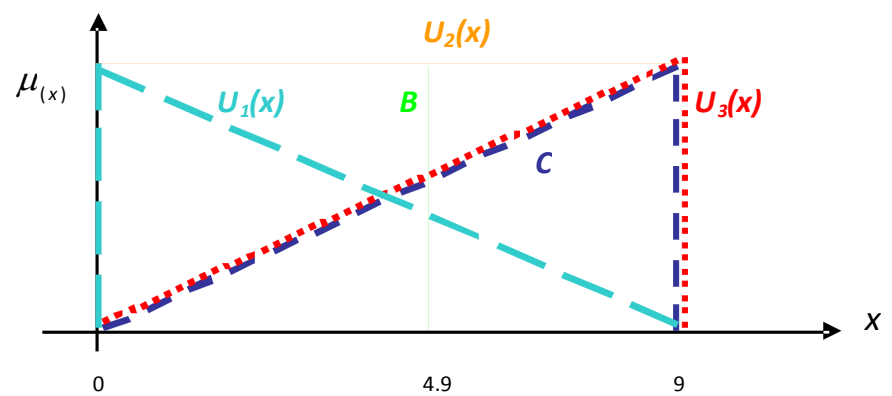

Fig. 6. Comparison of fuzzy numbers $B=(4.9,4.9,4.9)$ and $C=(0,9,9)$ based on descending, constant and ascending linear utilitv functions

The preference ratios are calculated using different linear utility functions as follows,

$$
\begin{array}{lll}
U_{1}(x): & R(B)=0.7925, & R(C)=0.2075===B>C \\
U_{2}(x): & R(B)=0.5444, & R(C)=0.4556===B>C \\
U_{3}(x): & R(B)=0.2964, & R(C)=0.7036===B<C
\end{array}
$$

\section{a.3. Pair-wise comparison between $A$ and $C$}

Finally, fuzzy numbers $\mathrm{A}$ and $\mathrm{C}$ are compared in Fig. 7. Turning point in the interval $[0,9]$ is 8.9503 . $\mathrm{C}$ is greater than $\mathrm{A}$ from 0 to 8.9503 and then, $\mathrm{A}$ is greater than $\mathrm{C}$. 


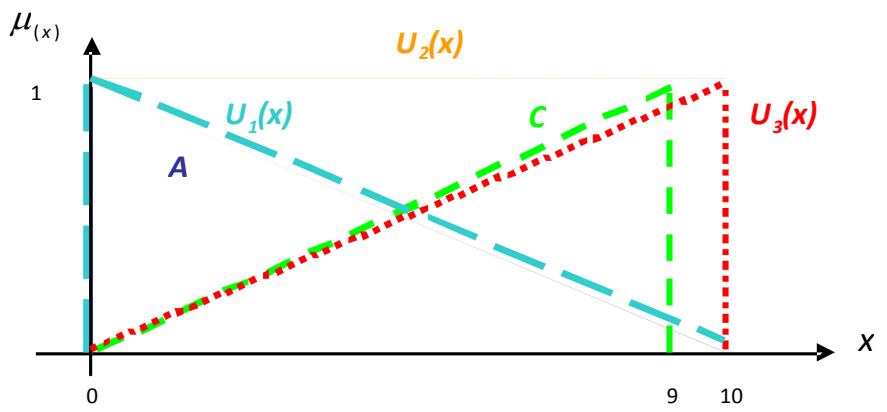

Fig. 7. Comparison of fuzzy numbers $A=(0,0,10)$ and $C=(0,9,9)$ based on descending, constant and ascending linear utility functions

The preference ratios are also calculated using different linear utility functions as follows,

$$
\begin{array}{lll}
U_{1}(x): & R(A)=0.0110, & R(C)=0.9890===>A<C \\
U_{2}(x): & R(A)=0.1050, R(C)=0.8950==>A<C \\
U_{3}(x): & R(A)=0.1989, \quad R(C)=0.8011===A<C
\end{array}
$$

According to this example, comparison of $A, B$ and $C$ based on descending linear utility function $U_{l \text { - }}$ (x) results in $A<B, B>C$ and $A<C$; then the ranking is $B>C>A$. If DM takes ascending linear utility function $U_{3}(x)$ into account, the results will change to $A>B, B<C$ and $A<C$; then the final ranking is $C>A>B$. But, if DM considers the utility of all points equally $\left(U_{2}(x)\right)$, the syllogism rule will not be held: $A>B, B>C$ and $A<C$.

b. In economic evaluation of projects, some values such as: invested values and their division types, projects' lifetime, sales (revenues) and costs (especially variable costs), may be uncertain. Since these values are vague, they can be considered as fuzzy. In a real case, in order to develop "Shabdiz Propylene Factory", two projects $A$ and $B$ including fuzzy costs have been suggested to DMs. The projects have been compared with each other using Fuzzy Net Present Worth method (FNPW method) and the following results have been obtained according to Table 3:

\section{Table 3}

FNPW of projects $A$ and $B$

\begin{tabular}{ll}
\hline Project & FNPW (Billion Rials) \\
\hline $\boldsymbol{A}$ & $(8,15,18)$ \\
$\boldsymbol{B}$ & $(10,12,20)$ \\
\hline
\end{tabular}

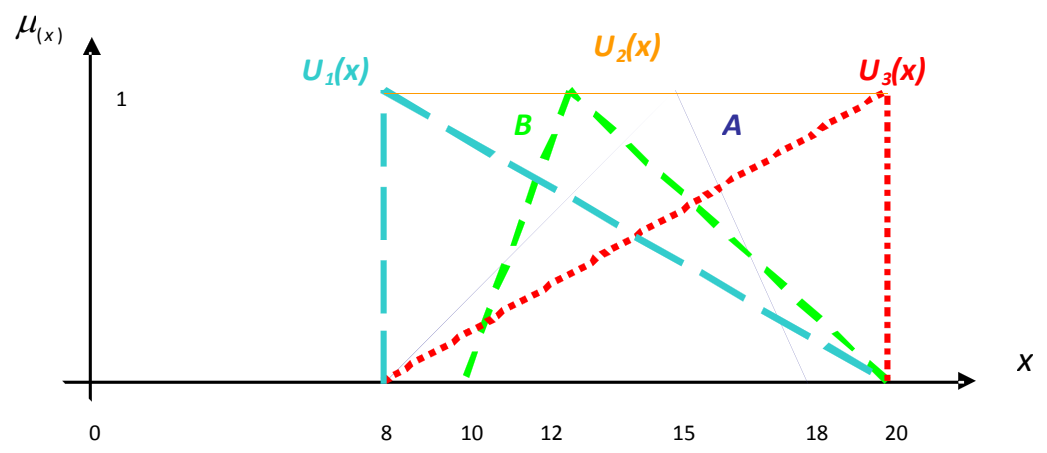

Fig. 8. Comparison of FNPW of projects A and B based on descending, constant and ascending linear utility functions 
Now, DMs must choose one of the projects. DMs cannot distinguish which project is more economic based on FNPWs. Therefore, they can rank FNPWs by applying "preference ratio based on utility function"' algorithm.

Fig. 8 displays projects A and B and utility functions $\mathrm{U1}(\mathrm{x}), \mathrm{U} 2(\mathrm{x})$ and $\mathrm{U} 3(\mathrm{x})$. There are two turning points: 12.37798 and 14.82202. The preference ratios based on U1(x), U2(x) and U3(x) are obtained as follows:

$$
\begin{array}{lll}
U_{1}(x): & R(A)=0.217248, & R(B)=0.782752===>A<B \\
U_{2}(x): & R(A)=0.203670, & R(B)=0.796330==>A<B \\
U_{3}(x): & R(A)=0.190092, & R(B)=0.809908===A<B
\end{array}
$$

The results show that project $B$ is more economic than project $A$ in all cases, but with different relational preference values. Thus, project $B$ is selected by DMs.

\section{Concluding remarks}

In this paper, a novel approach based on DM's utility using preference ratio concept was proposed to rank fuzzy numbers. The main contribution of this work is defining the value of DM's opinion for each point as a utility function. This function, which may be defined differently from the DMs' point of view, can be useful in evaluating fuzzy decisions. The results denoted the proposed method overcomes the shortcoming of preference ratio method. Also, the numerical examples showed how different utility functions can change the final ranking. At the end, this approach is recommended for MADM problems, expert systems and control in fuzzy environment. The proposed method can be directly applied to discrete fuzzy sets as well.

\section{Acknowledgment}

The authors would like to thank the anonymous referees for constructive comments on earlier version of this work.

\section{References}

Abbasbandy, S., \& Asady, B. (2006). Ranking of fuzzy numbers by sign distance. Information Science, 176(16), 2405-2416.

Abbasbandy, S., \& Hajjari, T. (2009). A new approach for ranking of trapezoidal fuzzy numbers. Computers and Mathematics with Applications, 57(3), 413-419.

Asady, B., \& Zendehnam, M. (2007). Ranking fuzzy numbers by distance minimization. Applied Mathematical Modeling, 31(11), 2589-2598.

Asady, B. (2010). The revised method of ranking LR fuzzy number based on deviation degree. Expert Systems with Applications, 37(7), 5056-5060.

Bortolan, G., \& Degani, R. (1985). A review of some methods for ranking fuzzy subsets. Fuzzy Sets and Systems, 15(1), 1-19.

Chen, S.H. (1985). Ranking fuzzy numbers with maximizing set and minimizing set. Fuzzy Sets and Systems, 17(2), 113-129.

Chen, S.J., \& Hwang, C.L. (1992). Fuzzy Multiple Attribute Decision Making. Springer, Berlin.

Chen, C.B., \& Klein, C.M. (1997). A simple approach to ranking a group of aggregated fuzzy utilities. IEEE Transaction on Systems Man and Cybernetics, 27(1), 26-35.

Chen, C.B., \& Klein, C.M. (1997). An efficient approach to solving fuzzy MADM problems. Fuzzy Sets and Systems, 88(1), 51-67.

Chen, C.C., \& Tang, H.C. (2008). Ranking nonnormal p-norm trapezoidal fuzzy numbers with integral value. Computers and Mathematics with Applications, 56(9), 2340-2346. 
Chen, S.M., \& Chen, J.H. (2009). Fuzzy risk analysis based on ranking generalized fuzzy numbers with different heights and different spreads. Expert Systems with Applications, 36(3), 6833-6842.

Chen, S.M., \& Wang, C.H. (2009). Fuzzy risk analysis based on ranking fuzzy numbers using $\alpha$-cuts, belief features and signal/noise ratios. Expert Systems with Applications, 36(3), 5576-5581.

Choobineh, F., \& Li, H. (1993). An index for ordering fuzzy numbers. Fuzzy Sets and Systems, 54(3), 287-294.

Chu, T., \& Tsao, C. (2002). Ranking fuzzy numbers with an area between the centroid point and original point. Computers and Mathematics with Applications, 43(1), 111-117.

Delgado, M., Verdegay, J.L., \& Villa, M.A. (1988). A procedure for ranking fuzzy numbers using fuzzy relations. Fuzzy Sets and Systems, 26(1), 49-62.

Fortemps, P., \& Roubens, M. (1996). Ranking and defuzzification methods based on area compensation. Fuzzy Sets and Systems, 82(3), 319-330.

Huijun, S., \& Jianjun, W. (2006). A new approach for ranking fuzzy numbers based on fuzzy simulation analysis method. Applied Mathematics and Computation, 174(1), 755-767.

Kim, K., \& Park, K.S. (1990). Ranking fuzzy numbers with index of optimism. Fuzzy Sets and Systems, 35(2), 143-150.

Lee, E.S., \& Li, R.J. (1988). Comparison of fuzzy numbers based on the probability measure of fuzzy events. Computers and Mathematics with Applications, 15(10), 887-896.

Lee, L.W., \& Chen, S.M. (2008). Fuzzy risk analysis based on fuzzy numbers with different shapes and different deviations. Expert Systems with Applications, 34(4), 2763-2771.

Liou, T.S., \& Wang, M.J. (1992). Ranking fuzzy numbers with integral value. Fuzzy Sets and Systems, 50(3), 247-255.

Liu, H.K., Wu, B., \& Liu, M.L. (2008). Investors' preference order of fuzzy numbers. Computers and Mathematics with Applications, 55(11), 2623-2630.

Ma, L.C., \& Li, H.L. (2008). A fuzzy ranking method with range reduction techniques. European Journal of Operational Research, 184(3), 1032-1043.

Mabuchi, G. (1988). An approach to the comparison of fuzzy subsets with an $\alpha$-cut dependent index. IEEE Transactions on Systems Man and Cybernetics, 18(2), 264-272.

Modarres, M., \& Sadi-Nezhad, S. (2001). Ranking fuzzy numbers by preference ratio. Fuzzy Sets and Systems, 118(3), 429-436.

Modarres, M., \& Sadi-Nezhad, S. (2005). Fuzzy simple additive weighting method by preference ratio. Intelligent Automation and Soft Computing, 11(4), 235-244.

Sadi-Nezhad, S., \& Ghaleh Assadi, R. (2008). Preference ratio-based maximum operator approximation and its application in fuzzy flow shop scheduling. Applied soft computing, 8(1), 759-766.

Sadi-Nezhad, S., \& Khalili Damghani, K. (2010). Application of a fuzzy TOPSIS method base on modified preference ratio and fuzzy distance measurement in assessment of traffic police centers performance. Applied soft computing, 10(4), 1028-1039.

Shieh, B.S. (2007). An approach to centroids of fuzzy numbers. International Journal of Fuzzy Systems, 9(1), 51-54.

Tseng, T.Y., \& Klein, C.M. (1989). New algorithm for the ranking procedure in fuzzy decision making. IEEE Transactions on System Man and Cybernetics, 19(5), 1289-1296.

Wang, Y.M., Yang, J.B., Xu, D.L., \& Chin, K.S. (2006). On the centroids of fuzzy numbers. Fuzzy Sets and Systems, 157(7), 919-926.

Wang, Y.J., \& Lee, S.H. (2008). The revised method of ranking fuzzy numbers with an area between the centroid and original points. Computers and Mathematics with Applications, 55(9), 2033-2042.

Wang, Y.M. (2009). Centroid defuzzification and the maximizing set and minimizing set ranking based on alpha level sets. Computers \& Industrial Engineering, 57(1), 228-236.

Wang, Y.M., \& Luo, Y. (2009). Area ranking of fuzzy numbers based on positive and negative ideal points. Computers and Mathematics with Applications, 58(9), 1769-1779. 
Wang, Z.X., Liu, Y.J., Fan, Z.P., \& Feng, B. (2009). Ranking LR fuzzy number based on deviation degree. Information Sciences, 179(13), 2070-2077.

Zadeh, L.A. (1965). Fuzzy sets. Information and Control, 8(3), 338-353. 